\title{
Reconocimiento y manejo de la Anafilaxia en pediatría
}

\author{
Detection and management of Anaphylaxis in children
}

\author{
Fustiñana Ana Laura ${ }^{\mathrm{a}}$, Rino Pedro B. ${ }^{\mathrm{a}}$, Kohn-Loncarica Guillermo A. ${ }^{\mathrm{a}}$
}

aUnidad de Emergencias del Hospital de Pediatría Prof. Dr. Juan P. Garrahan. Argentina.

Recibido el 17 de julio de 2018; aceptado el 23 de octubre de 2018

\section{Resumen}

Introducción: La anafilaxia es una emergencia. De acuerdo con las últimas recomendaciones internacionales el reconocimiento de los criterios clínicos y el tratamiento temprano con adrenalina intramuscular se asocian a mayor sobrevida. Objetivo: Determinar el conocimiento de los médicos pediatras de un Hospital Pediátrico de tercer nivel sobre los criterios diagnósticos y el tratamiento de la anafilaxia. Material y Método: Estudio descriptivo transversal que considera diseño, aplicación y validación de una encuesta anónima a médicos con residencia completa en pediatría que realizan guardias en un hospital de tercer nivel. Los ítems de la Encuesta comprendieron tres dimensiones, experiencia del operador (2 ítems), manejo farmacológico (3 ítems) e identificación del cuadro (4 ítems). El análisis estadístico utilizó el programa SPSS v.21, presentando medidas de tendencia central (mediana, rango y tabla de frecuencias) y para su comparación prueba de Chi cuadrado. Se consideró significativo un valor de $\mathrm{p}<0,05$. Resultados: Se encuestaron 71 médicos con una mediana de 3 años transcurridos desde el fin de la residencia. 35\% identificó todos los criterios clínicos; 99\% (70) indicó adrenalina, 73\% por vía intramuscular y 55\% a dosis correcta (solo el $48 \%$ contestó la dosis y vía correctamente). En forma global la adecuación para identificación más manejo correcto fue del $21 \%$. Los médicos con menos de 5 años de experiencia tuvieron mejor desempeño en la administración de adrenalina intramuscular $(83 \%$ vs $52 \%$ p = 0,005) y en la detección de síntomas gastrointestinales $(60 \% v s 35 \% \mathrm{p}=0,043)$. Conclusiones: Existen dificultades para la identificación y el manejo apropiado de la anafilaxia por pediatras de un Hospital de tercer nivel en un escenario teórico. Aunque la mayoría eligió la adrenalina como droga de primera línea, la mitad no la indicó de forma correcta y solo un tercio reconoció el cuadro en todos sus escenarios.

\section{Abstract}

Introduction: Anaphylaxis is an emergency condition. According to the latest international guidelines, early recognition and treatment with intramuscular epinephrine are associated with increased survival. Objective: To determine the level of knowledge of pediatricians in a tertiary Pediatric Hospital about the diagnostic criteria and treatment of anaphylaxis. Material and Method: A cross-sec-
Palabras clave:

Anafilaxia;

epinefrina;

tratamiento de

urgencia;

diagnóstico temprano 
tional descriptive study was conducted, designing, applying, and validating an anonymous survey to physicians with complete residency in pediatrics who are on call at a third level hospital. The statistical analysis was made using the SPSS v.21 software, presenting measures of central tendency (median, range, and frequency table) and Chi-square test for comparison. A value of $\mathrm{p}<0.05$ was considered significant. Results: 71 physicians completed the survey with a median of three years after the end of residency.35\% of them identified all clinical criteria, 99\% (70) indicated epinephrine, 73\% chose the intramuscular route, and 55\% indicated the correct dose. Only $48 \%$ of responders chose the dose and administration route correctly. In general, $21 \%$ recognized anaphylaxis and used epinephrine correctly. Physicians with less than five years of experience performed better in the intramuscular administration of epinephrine ( $83 \%$ vs $52 \% \mathrm{p}=0.005)$ and in the detection of gastrointestinal symptoms $(60 \%$ vs $35 \% \mathrm{p}=0.043)$. Conclusions: There are difficulties in the identification and proper management of anaphylaxis by pediatricians of a tertiary Pediatric Hospital in a theoretical clinical setting. Although most of pediatricians chose epinephrine as a first-line drug, half of them did not indicate it correctly, and only one-third recognized anaphylaxis in all scenarios.

\section{Introducción}

La anafilaxia es una urgencia cuyo reconocimiento y manejo temprano salva vidas. El reconocimiento de los criterios clínicos (tabla 1) definidos en el 2005 durante una reunión de expertos del Instituto Nacional de la Salud de EE. UU. (NIH, National Institutes of Health) $)^{(3)} \mathrm{y}$ la adrenalina intramuscular como droga de elección y primera acción de tratamiento resultan claves en la reversión de los síntomas según las últimas recomendaciones $^{(4-7)}$.

En los últimos 15 años se ha observado un aumento de la incidencia de la anafilaxia en EE. UU. y Europa ${ }^{(8,9)}$. Entre 2008 y 2014 en los servicios de emergencias (SE) pediátricas de EE. UU. la anafilaxia como motivo de consulta aumentó un $147 \%^{(10)}$, acompañándose de un incremento de las alergias alimentarias registradas en los niños entre 1997 y $2007^{(11)}$.

Los SE suelen constituir el primer lugar en la atención de la anafilaxia. Pese al incremento de los casos continúan existiendo dificultades en el reconocimiento y correcto manejo del cuadro ${ }^{(12-16)}$. Frente a esta realidad decidimos administrar una encuesta a médicos pediatras qué atienden emergencias en un Hospital Pediátrico de tercer nivel para evaluar el conocimiento sobre las últimas recomendaciones ${ }^{(4-7)}$ en el manejo y reconocimiento de la anafilaxia.

\section{Material y Método}

Estudio descriptivo de corte transversal, realizado a través del diseño y aplicación de una encuesta el 24 de agosto de 2016 en el Hospital de Pediatría "Prof. Dr. Juan P. Garrahan", hospital de tercer nivel (Ciudad Autónoma de Buenos Aires (CABA), Argentina), con un número aproximado de 90.000 consultas anuales en el Servicio de Emergencias (SE), 300 camas de Cuidados Intermedios y Moderados (CIM) y 534 camas totales. Población: médicos con residencia completa en pediatría que realizan guardias ${ }^{*}$ en los sectores de CIM y SE. Se excluyeron aquellos de licencia al momento de la encuesta y los médicos de planta ${ }^{* *}$ y becarios del SE. Se registró cantidad de años de desempeño como médico de guardia luego de haber completado la residencia de pediatría.

La encuesta (figura 1) fue auto administrada y anónima. Un mismo operador la entregó, presenció y recolectó una vez finalizado su llenado con el fin de evitar las consultas con otros colegas o a través de fuentes bibliográficas. La encuesta se elaboró en base a otras encuestas publicadas ${ }^{(17,18)}$ y fue adaptada a nuestro objetivo. El diseño estuvo a cargo de médicos senior del SE. A los fines de validar su comprensión se administró a cuatro médicos con experiencia en la atención de pacientes en situaciones de emergencia que no participaron del estudio y con sus sugerencias se llegó a la versión final (face validity) que consta de 9 ítems con modalidad pregunta de opción múltiple y respuesta corta. Los ítems comprenden tres dimensiones, experiencia del operador (2 ítems), manejo farmacológico (3 ítems) e identificación del cuadro (4 ítems). Se solicitó consentimiento informado.

El estudio se realizó un solo día durante 8 horas consecutivas a fin de evitar la divulgación del contenido. Se entregó en un sobre cerrado para garantizar el anonimato. El consentimiento se consignó en una hoja separada para asegurar la confidencialidad. A los fines de disminuir el sesgo de información y mejorar la confiabilidad del instrumento un solo operador fue el encargado de

* En el Hospital Juan P. Garrahan los turnos de guardia comprenden los horarios de 16 a 8 h los días hábiles y durante las 24 h los no hábiles. Durante este período el trabajo médico es realizado por los médicos de planta que una vez por semana extienden su horario.

** Médicos pediatras que realizan sus tareas de lunes a viernes de 8 a 16 hs en una Unidad o Servicio de la institución. 


\begin{tabular}{|c|c|c|c|c|}
\hline $\begin{array}{l}\text { Condición } \\
\text { clínica }\end{array}$ & Definición & Fisiopatología & Diagnóstico & Tratamiento primera línea \\
\hline Urticaria $^{(1)}$ & $\begin{array}{l}\text { Desarrollo de ronchas, } \\
\text { angioedema o ambos. }\end{array}$ & $\begin{array}{l}\text { Degranulación de } \\
\text { mastocitos }\end{array}$ & Clínico & $\begin{array}{l}\text { Bloqueantes histamínicos } \mathrm{H}_{1} \\
\text { de segunda generación }\end{array}$ \\
\hline $\begin{array}{l}\text { Angioedema } \\
\text { hereditario }^{(2)}\end{array}$ & $\begin{array}{l}\text { Reacción vascular } \\
\text { de la dermis o tejido } \\
\text { mucoso/submucoso } \\
\text { que resulta en edema } \\
\text { que puede ocasionar } \\
\text { asfixia. }\end{array}$ & $\begin{array}{l}\text { Reacción vascular } \\
\text { secundaria a la } \\
\text { producción de } \\
\text { bradiquininas por } \\
\text { déficit o disfunción } \\
\text { del inhibidor C1 } \\
\text { (C1-INH) }\end{array}$ & $\begin{array}{l}\text { Se sospecha: } \\
\text { 1. Historia familiar } \\
\text { 2. Comienzo en la niñez o la adolescencia } \\
\text { 3. Dolor abdominal recurrente } \\
\text { 4. Edema de tracto respiratorio superior } \\
\text { 5. Falta de respuesta frente a tratamiento } \\
\text { con antihistamínicos, corticoide o adrena- } \\
\text { lina } \\
\text { 6. Síntomas prodrómicos } \\
\text { 7. Ausencia de urticaria } \\
\text { Diagnóstico: Dosaje bajo de C4, dosaje o } \\
\text { disfunción de C1-INH }\end{array}$ & $\begin{array}{l}\text { Concentrado plasmático } \\
\text { C1-INH } \\
\text { C1- INH recombinante } \\
\text { Se debe hacer profilaxis } \\
\text { frente a procedimientos } \\
\text { (odontógenos, endoscó- } \\
\text { picos) }\end{array}$ \\
\hline Anafilaxia ${ }^{(3)}$ & $\begin{array}{l}\text { Reacción de } \\
\text { hipersensibilidad } \\
\text { aguda, que puede } \\
\text { comprometer la vida }\end{array}$ & $\begin{array}{l}\text { Reacción mediada } \\
\text { por lgE o por } \\
\text { inmunocomplejos }\end{array}$ & $\begin{array}{l}\text { Es muy probable cuando cumple uno de los } \\
3 \text { siguientes: } \\
\text { 1. Inicio agudo de una enfermedad (minutos } \\
\text { a pocas horas) que comprometa la piel, } \\
\text { mucosa o ambas. } \\
\text { Y al menos uno de los siguientes: } \\
\text { a. Compromiso respiratorio } \\
\text { b. Reducción de la presión arterial o sínto- } \\
\text { mas asociados a disfunción de órgano } \\
\text { (colapso, síncope, incontinencia, etc.) } \\
\text { 2. Dos o más de los siguientes que ocurran } \\
\text { de manera rápida tras la exposición a un } \\
\text { presunto alérgeno (minutos a pocas ho- } \\
\text { ras): } \\
\text { a. Compromiso de piel y/o mucosas } \\
\text { b. Compromiso respiratorio } \\
\text { c. Reducción de la presión arterial o sínto- } \\
\text { mas asociados a disfunción de órgano } \\
\text { (colapso, síncope, incontinencia, etc.) } \\
\text { d. Síntomas gastrointestinales persistentes } \\
\text { 3. Reducción de la presión arterial luego de } \\
\text { la exposición a un alérgeno conocido } \\
\text { a. Infantes y niños: TAS baja para la edad } 1 \\
\text { o disminución del } 30 \% \text { de la TAS } \\
\text { b. Adultos: TAS } 90 \text { mmHg o caída }>30 \% \\
\text { de la TAS habitual. }\end{array}$ & $\begin{array}{l}\text { Adrenalina } 1: 1.000 \text { a dosis } \\
0,01 \mathrm{mg} / \mathrm{kg} \text { (dosis máxima } \\
0,3-0,5 \mathrm{mg} \text { ) intramuscular } \\
\text { en cara lateral del muslo. } \\
\text { Posición supina y } \mathrm{O}_{2} \\
\text { Expansión con volumen si } \\
\text { existe compromiso hemo- } \\
\text { dinámico }\end{array}$ \\
\hline
\end{tabular}

TAS = tensión arterial sistólica. ${ }^{1}$ En niños TAS baja para la edad cuando: es $<$ a $70 \mathrm{mmHg}$ en niños de $1 \mathrm{mes}$ a 1 año o $<$ a $(70 \mathrm{mmHg}+($ edad $\chi^{2}$ ) en niños entre 1 a 10 años o < a 90 mmHg en niños entre 11 y 17 años.

1. ¿Cuantos años/meses lleva realizando guardias en la institución desde que concluyó su residencia?: años o meses

2. ¿Ha atendido alguna vez un episodio de anafilaxia severa/Shock anafiláctico? SI ( ) NO ( )

3. ¿Cuál es la medicación de primera línea que utiliza ante episodio de anafilaxia severa/ shock anafiláctico? (complete el espacio en blanco con una sola droga)

3. a) ¿Qué vía de administración usa?: VO ( ) IV ( ) IM ( ) SC ( ) (marque con una cruz la que corresponda) b) ¿Qué dosis utiliza? ( $\mathrm{mg} / \mathrm{kg})$ : ___ (complete el espacio en blanco)

4. Frente a cuál de los siguientes síntomas considera ud. que está ante una anafilaxia severa/shock anafiláctico. (marque con una cruz, puede dejar en blanco o seleccionar más de una opción)
A. Comienzo súbito de reacción cutánea urticariforme y broncoespasmo.
B. Comienzo súbito tras exposición a alérgeno de rash generalizado pruriginoso y vómitos incoercibles.
C. Episodio súbito de eritrodermia con prurito.
D. Episodio sincopal, con bradicardia en los minutos posteriores a la infusión IV de un fármaco.

Figura 1. Encuesta administrada a médicos que realizan guardias en los sectores de internación y emergencias. $\mathrm{VO}=$ vía oral, $\mathrm{IV}=$ vía intravenosa, $\mathrm{IM}=$ vía intramuscular, $\mathrm{SC}=$ vía subcutánea. 
administrar la encuesta. Para el análisis estadístico se utilizó el programa estadístico SPSS v.21, para variables numéricas y categóricas se utilizaron medidas de tendencia central (mediana, rango y tabla de frecuencias) y para su comparación prueba de Chi cuadrado. Un valor de $\mathrm{p}<0,05$ se consideró estadísticamente significativo.

\section{Resultados}

En el hospital de pediatría "Prof. Dr. Juan P. Garrahan", 100 médicos con residencia completa en pediatría realizan guardias en los sectores de CIM y SE. Se encuestaron 71 médicos y 29 se excluyeron (18 se encontraban de licencia, 3 no estaban disponibles, 5 eran médicos de planta y 3 becarios del SE), 67/71 respondieron la encuesta de manera completa. La mediana de años transcurridos desde el fin de la residencia fue de 3 años (0,1-24 años). El 69\% (49) refirió haber presenciado un episodio de anafilaxia alguna vez en su práctica diaria.

Se realizaron 4 preguntas para evaluar el conocimiento de los criterios diagnósticos publicados el 2005 por $\mathrm{NIH}^{(1)}$ (tabla 2) Solo el 35\% (25) reconoció todos los criterios de anafilaxia.

Si bien el 99\% (70) seleccionó a la adrenalina como droga de primera elección para el tratamiento (tabla 3) solo el 73\% (52) la indicó por la vía recomendada (intramuscular) y el 55\% (39) a la dosis correcta $(0,01$ $\mathrm{mg} / \mathrm{kg}$ ). Cuando analizamos de forma completa la indicación de adrenalina (elección, dosis y vía) observamos que 48\% (34) respondió correctamente.

$\mathrm{Al}$ analizar de forma global tanto el reconocimiento clínico de anafilaxia como la elección y utilización correcta de la adrenalina advertimos que solo el 21\% (15) de los encuestados respondieron adecuadamente.
Finalmente, cuando comparamos el manejo de la anafilaxia según la experiencia del encuestado (tabla 4) observamos que los que tienen menos de 5 años de experiencia reconocen con más frecuencia la asociación de síntomas gastrointestinales persistentes y síntomas mucocutáneos $(60 \%$ vs $35 \% \mathrm{p}=0,043)$ y administran la adrenalina de forma intramuscular con más asiduidad $(83 \%$ vs $52 \% \mathrm{p}=0,006)$.

Tabla 2. Reconocimiento de criterios clínicos de anafilaxia

\begin{tabular}{lc}
\hline Criterios clínicos & $\mathrm{n}=71(\%)$ \\
\hline Piel + Respiratorio & $60(85)$ \\
Piel + Gastrointestinal & $37(52)$ \\
Neurológico + cardiovascular & $60(85)$ \\
Piel* $^{*}$ & $10(14)$ \\
\hline
\end{tabular}

Criterios clínicos diagnósticos de anafilaxia según el Instituto Nacional de Salud de Estados Unidos. *El compromiso de piel aislado no es un criterio diagnóstico

Tabla 3. Dosis y vía de administración de la adrenalina

\begin{tabular}{llr}
\hline Adrenalina & & $\mathrm{n} 70,(\%)$ \\
\hline Vía de administración & IM & $52(74)$ \\
& $\mathrm{SC}$ & $5(7)$ \\
& $\mathrm{IV}$ & $13(19)$ \\
Dosis & $0,01 \mathrm{mg} / \mathrm{kg}$ & $39(56)$ \\
& $0,1 \mathrm{mg} / \mathrm{kg}$ & $25(36)$ \\
& $1 \mathrm{mg} / \mathrm{kg}$ & $2(3)$ \\
& $0,001 \mathrm{mg} / \mathrm{kg}$ & $1(1)$ \\
& $\mathrm{NC}$ & $3(4)$ \\
\hline
\end{tabular}

$\mathrm{IM}=$ intramuscular, $\mathrm{SC}=$ subcutánea, $\mathrm{IV}=$ intravenosa, $\mathrm{NC}=$ No contesta. Hubo un encuestado que no eligió la adrenalina como droga de primera línea.

Tabla 4. Comparación entre médicos con menos de 5 años de experiencia y aquellos con más de 5 años en el reconocimiento y manejo de anafilaxia

\begin{tabular}{|c|c|c|c|c|}
\hline \multicolumn{2}{|c|}{ Variables de reconocimiento y tratamiento } & \multirow{2}{*}{$\begin{array}{c}\begin{array}{c}\text { Experiencia < } 5 \text { años } \\
\text { n } 48,(\%)\end{array} \\
41(85)\end{array}$} & \multirow{2}{*}{$\begin{array}{c}\text { Experiencia > } 5 \text { años } \\
\text { n 23,(\%) }\end{array}$} & \multirow{2}{*}{$\begin{array}{c}p^{*} \\
0,76\end{array}$} \\
\hline Reconocimiento de & Respiratorio + Piel & & & \\
\hline los criterios clínicos & Piel + Gastrointestinal & $29(60)$ & $8(35)$ & 0,043 \\
\hline & SNC + Cardiovascular & $39(81)$ & $21(91)$ & 0,27 \\
\hline & Piel aislada** & $8(17)$ & $2(9)$ & 0,37 \\
\hline & Reconoce todos los criterios & $20(42)$ & $5(22)$ & 0,1 \\
\hline \multirow{3}{*}{$\begin{array}{l}\text { Tratamiento } \\
\text { con adrenlina } \\
\text { n (70) }\end{array}$} & Adrenalina IM & $40(83)$ & $12(52)$ & 0,006 \\
\hline & Dosis adrenalina $0,01 \mathrm{mg} / \mathrm{kg}$ & $31(65)$ & $8(35)$ & 0,1 \\
\hline & Indicación correcta (adrenalina IM + 0,01 mg/kg) & $28(58)$ & $6(26)$ & 0,024 \\
\hline
\end{tabular}

Criterios clínicos NIH: Piel= síntomas mucocutáneos, Respiratorio= síntomas respiratorios, Gastrointestinal= síntomas gastrointestinales persistentes, SNC= síntomas neurológicos, Cardiovasculares= Síntomas cardiovasculares. Adrenalina IM= Indicación de adrenalina por vía intramuscular. Indicación correcta de adrenalina= adrenalina a 0,01 mg/kg por vía intramuscular. ${ }^{\star} p=$ prueba de chi2. ${ }^{*}$ No es un criterio de NIH. 
Tabla 5. Resumen de encuestas realizadas sobre reconocimiento y tratamiento de la anafilaxia.

\begin{tabular}{|c|c|c|c|c|c|c|c|c|}
\hline \multirow[t]{2}{*}{ Publicación } & \multirow[t]{2}{*}{ Población/Modalidad¹ } & \multicolumn{3}{|c|}{ Administración adrenalina ${ }^{2}$} & \multicolumn{4}{|c|}{ Reconocimiento criterios clínicos ${ }^{3}$} \\
\hline & & $\begin{array}{l}\text { AD } 1^{\text {ra }} \\
\%\end{array}$ & $\begin{array}{l}\mathrm{IM} \\
\%\end{array}$ & $\%$ & $\begin{array}{c}\text { Piel }+ \\
\text { Mucosas } \\
\%\end{array}$ & $\begin{array}{c}\text { Piel + Resp } \\
\%\end{array}$ & $\begin{array}{c}\text { Piel + } \\
\text { HipoTA } \\
\%\end{array}$ & $\begin{array}{c}\text { HipoTA + } \\
\text { Alérgeno } \\
\%\end{array}$ \\
\hline $\begin{array}{l}\text { Jose et al } \\
2007^{(19)}\end{array}$ & $\begin{array}{l}95 \\
\text { M residentes } \\
\text { presenciada }\end{array}$ & 94 & 58 & - & - & - & - & - \\
\hline $\begin{array}{l}\text { Grossman et al } \\
2013^{(18)}\end{array}$ & $\begin{array}{l}620 \\
\text { MEP } \\
\text { web }\end{array}$ & 94 & 67 & - & - & - & - & - \\
\hline $\begin{array}{l}\text { Baççioĝlu et al } \\
2013^{(20)}\end{array}$ & $\begin{array}{l}1172 \\
\text { M, E, Est M, PM } \\
\text { Correo electrónico }\end{array}$ & 45 & 29 & 29 & - & - & - & - \\
\hline $\begin{array}{l}\text { De Solé et al } \\
2013^{(21)}\end{array}$ & $\begin{array}{l}350 \mathrm{~A} \\
160 \mathrm{M} \text { no A } \\
\text { web }\end{array}$ & $\begin{array}{c}70 \\
(\mathrm{M} \text { no } \mathrm{A}) \\
90(\mathrm{~A})\end{array}$ & $\begin{array}{c}24 \\
(\mathrm{M} \text { no } \mathrm{A}) \\
78(\mathrm{~A})\end{array}$ & - & - & - & - & - \\
\hline $\begin{array}{l}\text { Ibrahim et al } \\
2014^{(22)}\end{array}$ & $\begin{array}{l}190 \\
\text { E,M } \\
\text { presenciada y anó- } \\
\text { nima }\end{array}$ & $\begin{array}{c}\mathrm{T}: 53 \\
\mathrm{M}(89) \\
\mathrm{E}(40)\end{array}$ & $\begin{array}{l}T: 57 \\
M(85) \\
E(47)\end{array}$ & $\begin{array}{c}T: 58 \\
M(73) \\
E(50)\end{array}$ & $\begin{array}{c}\mathrm{T}: 73 \\
\mathrm{M}(42) \\
\mathrm{E}(84)\end{array}$ & $\begin{array}{c}\text { T: } 89 \\
\text { M (94) } \\
\text { E (87) }\end{array}$ & $\begin{array}{c}\text { T: } 93 \\
\text { M (98) } \\
\text { E (91) }\end{array}$ & $\begin{array}{c}\mathrm{T}: 85 \\
\mathrm{M}(93) \\
\mathrm{E}(82)\end{array}$ \\
\hline $\begin{array}{l}\text { Wang et al } \\
2014^{(12)}\end{array}$ & $\begin{array}{l}7822 \\
\text { Socios Medscape } \\
\text { web }\end{array}$ & 95 & - & - & 5 & 85 & - & 57 \\
\hline $\begin{array}{l}\text { Plumb et al } \\
2015^{(23)}\end{array}$ & $\begin{array}{l}68 \\
\text { M recién graduados } \\
\text { presenciada anónima }\end{array}$ & 100 & 74 & - & 21 & 100 & - & - \\
\hline $\begin{array}{l}\text { Altman et al } \\
2105^{(24)}\end{array}$ & $\begin{array}{l}318 \\
\text { M } \\
\text { telefónica }\end{array}$ & $81-98$ & - & - & - & - & - & - \\
\hline $\begin{array}{l}\text { Colleti Junior et al } \\
2016^{(17)}\end{array}$ & $\begin{array}{l}43 \\
\text { M intensivista } \\
\text { presenciada }\end{array}$ & 84 & 42 & - & - & - & - & - \\
\hline $\begin{array}{l}\text { Drupad et al } \\
2015^{(25)}\end{array}$ & $\begin{array}{l}265 \\
\text { Est. M } \\
\text { Est. E } \\
\text { M internista }\end{array}$ & 57 & 16,5 & 26 & - & - & - & - \\
\hline
\end{tabular}

1) En población: $M=$ médico, $E=$ enfermero, Est= estudiante, $A=$ alergista, $M E P=$ médico emergentólogo pediátrico, $P M=$ paramédico. 2) En administración de adrenalina: AD $1^{\text {ra }}=$ Elección adrenalina como droga de primera línea; T= porcentaje total; IM= Indicación adrenalina por vía intramuscular; Dosis = Dosis correcta $(0,01 \mathrm{mg} / \mathrm{kg})$ de adrenalina. 3) En criterios clínicos: Piel + Mucosas= Síntomas concomitantes en piel y mucosas (no es un criterio $\mathrm{NIH}$ ); Piel + resp = Síntomas concomitantes en piel y/o mucosas y respiratorios (criterio $1 \mathrm{NIH}$ ); Piel + Hipo$\mathrm{TA}=$ Síntomas concomitantes en piel y/o mucosas más hipotensión arterial (criterio $2 \mathrm{NIH}$ ); HipoTA + alérgeno= Hipotensión arterial frente a exposición a un alérgeno (criterio $3 \mathrm{NIH}$ ).

\section{Discusión}

Un estudio publicado por Campbell et al. ${ }^{(26)}$ en el 2012 demostró que los criterios diagnósticos establecidos desde el 2005 por el Instituto de Alergias y Enfermedades Infecciosas y la Red de Alergias Alimentarias y Anafilaxia (NIAID/FAAN, National Institute of Allergy and Infectious Disease and the Food Allergy and Anaphylaxis Network) tienen una sensibilidad diagnóstica del $96,5 \%$ y una especificidad del $82,4 \%$. Sin embargo, las brechas en el reconocimiento de la anafilaxia sigue siendo hoy un problema mundial.
Actualmente, la Clasificación Internacional de Enfermedades de la Organización Mundial de la Salud (CIE-9 y CIE 10) para anafilaxia genera confusión entre los usuarios dado que considera a la hipotensión arterial o el shock en su definición y no incluye otros criterios del NIH, contribuyendo de esta manera al subdiagnóstico ${ }^{(27,28)}$. Posiblemente esta situación se modifique pronto a partir de la publicación del CIE-11 que posee una nueva sección de "Condiciones alérgicas y de hipersensibilidad" dentro del capítulo de "Desórdenes del sistema inmune" que reconoce por primera vez a la anafilaxia como una condición clínica ${ }^{(29,30)}$. 
Otro obstáculo frecuente en el reconocimiento es utilizar como sinónimos el shock anafiláctico y la anafilaxia. Hay que tener presente que la hipotensión arterial en niños es infrecuente como lo demuestran estudios retrospectivos en SE pediátricos. Alvarez-Perea et al. ${ }^{(13)}$ describieron una serie de 133 niños con anafilaxia en donde solo el 7\% presentó shock, Goetz et al. ${ }^{(14)}$ observaron que solo el $2 \%$ presentó hipotensión arterial asociada a otros síntomas y ninguno hipotensión arterial aislada asociada a alérgeno (criterio 3 de NIH), entre 211 niños con anafilaxia.

La mayoría de las encuestas realizadas a nivel mundial (tabla 5) hace hincapié en la elección y administración correcta de la adrenalina, y no tanto en el reconocimiento del cuadro que resulta el disparador para administrarla.

Nuestros resultados coincidentemente con las encuestas realizadas por Wang y et al. ${ }^{(12)} \mathrm{y}$ Jacobsen y et al. ${ }^{(31)}$, mostraron que las presentaciones clásicas con compromiso de piel y/o mucosa, síntomas respiratorios y shock fueron reconocidos con mayor facilidad que aquellos casos menos frecuentes con compromiso gastrointestinal .Cuando no hay compromiso de piel $(10-20 \%)^{(32)}$, Jacobsen y et al. ${ }^{(31)}$ y Wang y et al. ${ }^{(12)}$ observaron que solo el 3\% y el 50\% de los encuestados, respectivamente, reconocieron el cuadro.

Revisando historias clínicas de niños atendidos en el SE, Álvarez Perea y et al. ${ }^{(13)}$ encontraron que la anafilaxia fue reconocida solo en el 53\% de los casos. El resto de los pacientes ingresó con diagnóstico de urticaria, angioedema, reacción alérgica y no recibió el tratamiento adecuado.

El uso de una definición estandarizada, clave en el reconocimiento, es escaso. En Brasil, Russell et al. ${ }^{(33)}$ reportó que el 90\% de los SE no utilizan criterios estandarizados.

Las numerosas barreras existentes para una adecuada y oportuna identificación de la anafilaxia continúan representando una preocupación universal para la salud pública. Los resultados observados en nuestro trabajo refuerzan la necesidad de incrementar todas la medidas necesarias tendientes a mejorar la difusión de los criterios clínicos que permitan mejorar el reconocimiento de la entidad y dar paso al tratamiento.

Otro de los aspectos evaluados en la encuesta fue el tratamiento de primera línea recomendado internacionalmente ${ }^{(4-7)}$. Cuando comparamos nuestros resultados en relación a la elección de adrenalina como droga de primera línea con otras encuestas realizadas a médicos (tabla 5) los datos obtenidos son similares (entre $85-95 \%)$. Pero cuando observamos que sucede respecto al manejo del cuadro en función de los datos aportados por estudios retrospectivos en SE pediátricos Álvarez-Perea y et al. ${ }^{(13)}$, Goetz y et al. ${ }^{(14)}$, Wright y et al. ${ }^{(15)}$ y Robinson y et al. ${ }^{(16)}$ refirieren que la adrenalina se administra solo entre el $32 \%$ y el $68 \%$ de los episodios de anafilaxia en niños. Por otra parte, Álvarez-Perea et al. ${ }^{(13)} \mathrm{y}$ Wright y et al. ${ }^{(15)}$ comunicaron una alta utilización de corticoides ( $81 \%$ y $51 \%$, respectivamente) y bloqueantes histamínicos $(63 \%$ y $62 \%$, respectivamente) como drogas de primera elección. Wright et al. ${ }^{(15)}$ justificaron esta discrepancia por la falta de protocolos para el manejo de anafilaxia en los SE, bajo conocimiento de las guías de tratamiento y errores conceptuales sobre la seguridad de la adrenalina intramuscular en el tratamiento de la misma.

La selección de la vía intramuscular (73\%), y la dosis de $0,01 \mathrm{mg} / \mathrm{kg}(55 \%)$ en nuestro estudio fue similar, y en algunos casos mayor, que en las encuestas analizadas (tabla 5).

Pero advertimos que en nuestros resultados el 18\% eligió la vía intravenosa y 35\% dosis superiores a las recomendadas. Cardona et al. ${ }^{(34)}$ describieron que la administración de la adrenalina intravenosa y la utilización de dosis mayores a las recomendadas se asociaron a mayor frecuencia de efectos adversos pero no observaron mayor morbimortalidad.

Por último decidimos evaluar si la experiencia del profesional tiene relación con el reconocimiento adecuado y un tratamiento acorde a las últimas recomendaciones $^{(4-7)}$. Al igual que Coletti et al. ${ }^{(17)}$ hallamos que los médicos con menos años de experiencia indican en mayor proporción adrenalina intramuscular. En cambio Grossman et al. ${ }^{(18)}$ no encontraron asociación con la experiencia pero si en aquellos que habían realizado programas de residencia. Nuestro análisis también refleja que los médicos con menos años de experiencia reconocieron mejor los síntomas gastrointestinales. Estas diferencias podrían deberse a aumento del número de publicaciones sobre el tema en los últimos años ${ }^{(30)}$, a un entrenamiento más reciente, a la incorporación del tema en los últimos congresos de emergencia de la región y en el plan de estudios de los residentes.

Existen ciertas limitaciones en nuestro estudio; las encuestas no siempre reflejan el accionar frente a situaciones reales y las respuestas a encuestas de opción múltiple sobre un tema específico pueden resultar sesgadas. Sin embargo, los resultados de la encuesta nos han permitido tomar medidas de educación y entrenamiento dirigidas al personal de salud de la institución.

\section{Conclusión}

Existen dificultades para la identificación y el manejo apropiado de la anafilaxia por pediatras de un hospital de tercer nivel en un escenario teórico. Solamente un tercio de los encuestados (35\%) pudo reconocer todos los criterios clínicos de anafilaxia, que podría implicar la existencia de subdiagnóstico de esta 
entidad. Aunque la mayoría de los encuestados eligió a la adrenalina como droga de primera línea, más de la mitad la utilizó de manera incorrecta, con los riesgos que esto conlleva (subtratamiento y/o efectos adversos). Finalmente solo el 21\% identificó y trató adecuadamente el cuadro. Estos resultados sugieren la elaboración de estrategias educativas y protocolos de atención clínica que permitan optimizar el manejo de este tipo de eventos que requieren acciones urgentes.

\section{Responsabilidades éticas}

Protección de personas y animales: Los autores declaran que los procedimientos seguidos se conformaron a las normas éticas del comité de experimentación humana responsable y de acuerdo con la Asociación Médica Mundial y la Declaración de Helsinki.

Confidencialidad de los datos: Los autores declaran que han seguido los protocolos de su centro de trabajo sobre la publicación de datos de pacientes.

Derecho a la privacidad y consentimiento informado: Los autores han obtenido el consentimiento informado de los pacientes y/o sujetos referidos en el artículo. Este documento obra en poder del autor de correspondencia.

\section{Conflicto de intereses}

Los autores declaran no tener conflicto de intereses.

\section{Agradecimientos}

Agradecemos la colaboración de la Dra. Susana Rodriguez, Directora de Docencia e Investigación del Hospital "Dr. Prof. Juan P. Garrahan", por sus aportes y el tiempo dedicado que han contribuido a mejorar la calidad del manuscrito.

\section{Referencias}

1. Zuberbier T, Aberer W, Asero R, et al. The EAACI/GA(2) LEN/EDF/WAO Guideline for the definition, classification, diagnosis, and management of urticaria: the 2013 revision and update. Allergy. 2014;69:868-87.

2. Maurer M, Magerl M, Ansotegui I, et al. The international WAO/EAACI guideline for the management of hereditary angioedema - The 2017revision and update. Allergy. 2018; 73:1575-96.

3. Sampson HA, Muñoz-Furlong A, Campbell RL, et al. Second symposium on the definition management of anaphylaxis: summary report-Second National Institute of and Infectious Disease/Food Allergy Anaphylaxis Network symposium. J Allergy Clin Immunol. 2006;117:391-7.

4. Muraro A, Roberts G, Worm M, et al. Anaphylaxis: guidelines from the European Academy of Allergy and Clinical Immunology. Allergy. 2014;69:1026-45.

5. Lieberman P, Nicklas RA, Randolph C, Oppenheimer J, Bernstein D, Bernstein J. Anaphylaxis: a practice parameter update 2015. Annals of Allergy, Asthma \& Immunology. 2015;115:341-84.

6. Campbell RL, Li J, Nicklas RA, Sadosty $\mathrm{M}$. Emergency department diagnosis and treatment of anaphylaxis. 2013. Ann Allergy Asthma Immunol. 2014;113:599608.

7. Comité Nacional de Alergia. Actualización en las recomendaciones del tratamiento del choque anafiláctico: novedades sobre el uso de adrenalina. Arch Argent Pediatr. 2015;113:81-7.

8. Moneret-Vautrin DA, Morisset M, Flabbee J, Beaudouin E, Kanny G. Epidemiology of life-threatening and lethal anaphylaxis: a review. Allergy. 2005; 60:443-51.

9. Panesar SS, Javad S, de Silva D, et al. The epidemiology of anaphylaxis in Europe: a systematic review. Allergy. 2013;68:13531361.

10. Michelson KA, Hudgins JD, Burke LG, et al. Trends in Severe Pediatric Emergency Conditions in a National Cohort, 2008 to 2014. Pediatr Emer Care 2018;00:00-00.

11. Branum AM, Lukacs SL. Food Allergy Among Children in the United States. Pediatrics. 2009;124:1549-55.

12. Wang J, Young MC, Nowak-Wegrzyn A. International survey of knowledge of food-induced anaphylaxis. Pediatr Allergi Immunol. 2104;25:644-50.

13. Alvarez-Perea A, Ameiro B, Morales C, et al. Anaphylaxis in the Pediatric Emergency Department: Anaphylaxis of 133 Cases After and Allergy Workup. J Allergy Clin Inmunol Pract. 2017;5:12561263.

14. Goetz VL, Kim K, Stang AS. Pediatric anaphylaxis in the Emergency Department. Clinical Presentation, Quality of care, and Reliability of Consensus Criteria. Pediatr Emer Care. 2017;11:00-00.

15. Wright CD, Longiohn M, Lieberman PL, Lieberman JA. An analysis of anaphylaxis cases at a single pediatric emergency department during a 1-year period. Ann
Allergy Asthma Immunol. 2017;118:4614.

16. Robinson M, Greenhawt M, Stukus DR. Factors Associated with epinephrine administration for anaphylaxis in children before arrival to the emergency department: Ann Allergy Asthma Immonol. 2017;119:164-9.

17. Colleti J, de Carvalho WB. Anaphylaxis Knowledge Among Pediatric Intensivists in Brazil: A Multicenter Survey. J Intensive Care Med. 2017;32:593-6.

18. Grossman SL, Baumann BM, García Peña BM, Linares MY, Greenberg B, Hernandez-Trujillo VP. Anaphylaxis knowledge and practice of pediatric emergency medicine physicians: A national survey. J Pediatr. 2013;163: 841-6.

19. Jose R, Clesham GJ. Survey of the use of epinephrine (adrenaline) for anaphylaxis by junior hospital doctors. Postgrad Med J. 2007;83:610-611.

20. Baççıŏlu A, Yılmazel Uçar E. Level of knowledge about anaphylaxis among health care providers. Tuberk Toraks 2013;61:140-6.

21. Sole D, Ivancevich JC, Cardona V. Knowledge of anaphylaxis among IberoAmerican physicians: Results of the IberoAmerican online survey for physicians on the management and treatment of anaphylaxis (IOSPTA)- Latin American Society of Allergy, Asthma \& Immunology (LASAAI). J Investig Allergol Clin Immunol 2013;23:441-3.

22. Ibrahim I, Chew BL, Zaw WW, Van Bever HP. Knowledge of anaphylaxis among emergency department staff. Asia Pac 
Allergy. 2014;4:164-71.

23. Plumb B, Bright P, Gompels MM, Unsworth DJ. Correct recognition and management of anaphylaxis: not much change over a decade. Postgrad Med J. 2015;91:3-7.

24. Altman A, Camargo CA Jr, Simons FE, et al. Anaphylaxis in America: A national physician survey. J Allergy Immunol. 2015;135:830-3.

25. Drupad HS, Nagabushan H. Level of knowledge about anaphylaxis and its management among health care providers. Indian J Crit Care Med. 2015;19:412-5.

26. Campbell RL, Hagan JB, Manivannan $\mathrm{V}$, et al. Evaluation of National Institute of Allergy and Infectious Diseases/ Food Allergy and Anaphylaxis Network criteria for the diagnosis of anaphylaxis in emergency department patients. J. Allergy
Clin Inmunol. 2012;129:748-52.

27. Simons FE, Sampson HA. Anaphylaxis epidemic: Fact or fiction? J Allergy Clin Immunol. 2008;122:1166-8.

28. Tanno LK, Ganem F, Demoly P, Toscano CM, Bierrenbach AL. Undernotification of anaphylaxis deaths in Brazil due to difficult coding under the ICD-10. Allergy 2012;67:783-9.

29. Kase Tanno L, Bierrenbach AL, Simons FER, et al. Critical view of anaphylaxis epidemiology: open questions and new perspectives. Allergy Asthma Clin Immunol. 2018;14:12

30. Kase Tanno L, Chalmers RJG, Calderón MA, Aymé S, Demoly P, on behalf the Joint Allergy Academies (2017) Reaching multidisciplinary consensus on classification of anaphylaxis for the eleventh revision of the World Health Organization's (WHO) International
Classification of Diseases (ICD-11). Orphanet J Rare Dis. 2017; 12: 53.

31. Jacobsen RC, Toy S, Bonham AJ, Salomone JA 3rd, Ruthstrom J, Gratton M. Anaphylaxis knowledge among paramedics: results of a national survey. Prehosp Emerg Care. 2012;16:527-34.

32. Lieberman P, Nicklas RA, Oppenheimer $\mathrm{J}$, et al. The diagnosis and management of anaphylaxis practice parameter: 2010 Update. J Allergy Clin Immunol. 2010;126:477-80.

33. Russell SW, Farrar JR, Nowak R, et al. Evaluating the management of anaphylaxis in US emergency departments: Guidelines vs. practice. World J Emerg Med 2013; 4:98-106.

34. Cardona V, Ferré-Ybarz L, Guilarte $\mathrm{M}$, et al. Safety of Adrenaline Use in Anaphylaxis: A multicentre Register. Int Arch Allergy Immunol. 2017;173:171-7. 